DOI: $10.15393 /$ j3.art.2017.3871

UDC 517.518

E. S. Belkina, Yu. V. Malykhin

\title{
FOURIER COEFFICIENTS OF CONTINUOUS FUNCTIONS WITH RESPECT TO LOCALIZED HAAR SYSTEM
}

\begin{abstract}
We construct a nontrivial example of a continuous function $f^{*}$ on $[0,1]^{2}$ which is orthogonal to tensor products of Haar functions supported on intervals of the same length. This example clarifies the possible behaviour of Fourier coefficients of continuous functions with respect to a localized Haar system. The function $f^{*}$ has fractal structure. We give lower bounds on its smoothness.
\end{abstract}

Key words: fractals, Haar system, Haar wavelets

2010 Mathematical Subject Classification: 26B35, 42C10

1. Introduction. Let $\Delta_{k, i}=\left(\frac{i-1}{2^{k}}, \frac{i}{2^{k}}\right)$; by $\Delta_{k, i}^{+}$and $\Delta_{k, i}^{-}$we denote the left and right halves of $\Delta_{k, i}$, correspondingly.

Define the localized Haar system on $[0,1]^{2}$ in the following way (see fig. 1$): \chi_{0}(x, y) \equiv 1$ on $[0,1]^{2}$; other functions of the system are grouped into packs; for $k=0,1,2, \ldots$ the $k$-th pack consists of the functions

$$
\chi_{k, i, j}^{(1)}(x, y)= \begin{cases}2^{k}, & x \in \Delta_{k, i}^{+}, y \in \Delta_{k, j}, \\ -2^{k}, & x \in \Delta_{k, i}^{-}, y \in \Delta_{k, j}, \\ 0, & (x, y) \notin \overline{\Delta_{k, i}} \times \overline{\Delta_{k, j}},\end{cases}
$$

$$
\chi_{k, i, j}^{(2)}(x, y)=\chi_{k, j, i}^{(1)}(y, x), \quad \chi_{k, i, j}^{(3)}(x, y)=2^{-k} \chi_{k, i, j}^{(1)}(x, y) \chi_{k, i, j}^{(2)}(x, y),
$$

where $i, j=1,2, \ldots, 2^{k}$. (The values on the boundary of $\Delta_{k, i} \times \Delta_{k, j}$ are not important for us.) 

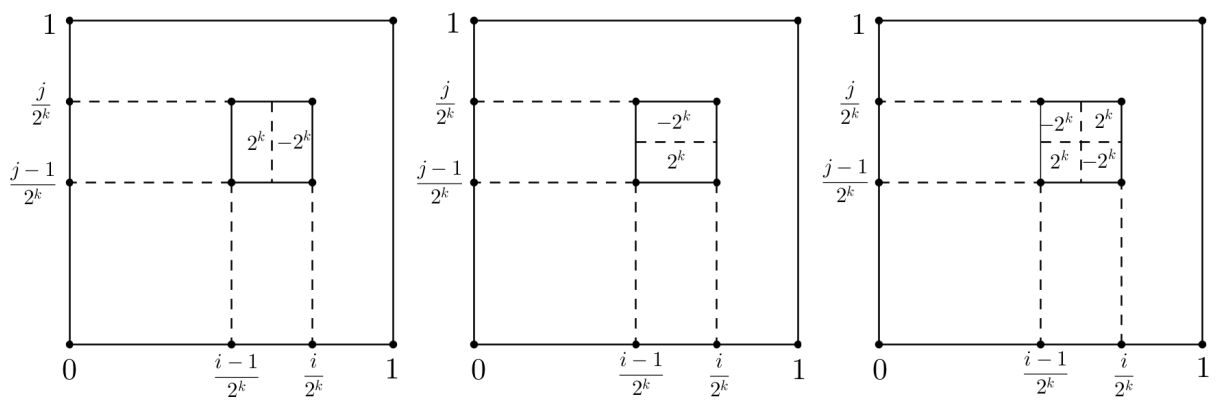

Fig. 1. Localized Haar system

We should note that the localized Haar system comes from the general construction of multivariate wavelets. Suppose that $\left\{V_{k}\right\}$ is a multiresolution analysis (MRA; see [2, Sect. 1.2]) in $L_{2}(\mathbb{R})$ with scaling function $\varphi_{0} \in V_{0}$. Denote by $W_{k}$ the orthogonal complement to $V_{k}$ in $V_{k+1}$. MRA generates the wavelet function $\psi \in W_{0}$ and its dyadic dilations and shifts $\left\{\psi_{k, i}\right\}$ form a basis of $L_{2}(\mathbb{R})$. There are at least two ways how to construct multivariate wavelets from univariate ones.

- The first way is to take the tensor product of one-dimensional wavelet functions: $\Psi_{K, I}:=\psi_{k_{1}, i_{1}}^{(1)} \otimes \psi_{k_{2}, i_{2}}^{(2)}$. The disadvantage of this method is that the new system is not "localized" even if the one-dimensional wavelets were: functions with large $K$ may have large support.

- The second way is to take the tensor product of the one-dimensional MRA's: $V_{k}=V_{k}^{(1)} \otimes V_{k}^{(2)}$. Then we define wavelet spaces $W_{k}$ by $V_{k+1}=V_{k} \oplus W_{k}$ and take basis in $W_{k}$.

The localized Haar system is obtained using the tensor product of simplest MRA with the scaling function $\varphi=\mathbf{1}_{[0,1)}$ and the wavelet function $\psi=\mathbf{1}_{[0,1 / 2)}-\mathbf{1}_{[1 / 2,1)}$. Three functions $\psi^{(1)}=\psi \otimes \varphi, \psi^{(2)}=\varphi \otimes \psi$ and $\psi^{(3)}=\psi \otimes \psi$ form a basis of $W_{0}$. Dyadic dilations and shifts of that functions (inside $[0,1]^{2}$ ) produce a localized Haar system, which is an orthonormal basis in $L^{2}[0,1]^{2}$ (see also [2, Sect. 2.8]).

For any summable function $f:[0,1]^{2} \rightarrow \mathbb{R}$ one can consider its FourierHaar coefficients:

$$
c_{k, i, j}^{(p)}(f)=\iint_{[0,1]^{2}} f(x, y) \chi_{k, i, j}^{(p)}(x, y) d x d y, \quad p=1,2,3 .
$$

It was observed by B. I. Golubov (see, e.g., [1, Chapter 3, §2]) that for a classical one-dimensional Haar system $\left\{\chi_{k, i}\right\}$ and for any $f \in C[0,1]$, 
$f \not \equiv$ const, one has

$$
\varlimsup_{k \rightarrow \infty} 2^{3 k / 2} \max _{i}\left|c_{k, i}(f)\right|>0,
$$

where $c_{k, i}(f)=\int_{0}^{1} f(x) \chi_{k, i}(x) d x$.

What happens on the square? If $f(x, y)=g(x)+h(y)$, everything reduces to the one-dimensional case. Indeed, $c_{k, i, j}^{(1)}(f)=2^{-k / 2} c_{k, i}(g)$, $c_{k, i, j}^{(2)}(f)=2^{-k / 2} c_{k, j}(h)$ and $c_{k, i, j}^{(3)}(f)=0$. So we shall not consider this trivial situation. In the general case, P. V. Glebov in his diploma work had proven the following.

Theorem A. If $f$ is a continuous function on $[0,1]^{2}$ and it is not represented as $f(x, y)=g(x)+h(y)$, then

$$
\varlimsup_{k \rightarrow \infty} 4^{k} \max _{i, j}\left|c_{k, i, j}^{(p)}\right|>0, \quad p=1,2 .
$$

We reproduce the proof here; it is rather short.

Proof. Suppose, $f \in C[0,1]^{2}$ and $\lim _{k \rightarrow \infty} 4^{k} \max _{i, j}\left|c_{k, i, j}^{(1)}\right|=0$; we shall prove that $f$ does not depend on $x$. Consider the "vertical average" function $f^{\circ}(x):=\int_{0}^{1} f(x, y) d y$. It is easy to compute that

$$
c_{k, i}\left(f^{\circ}\right)=2^{-k / 2} \sum_{j=1}^{2^{k}} c_{k, i, j}^{(1)}(f) .
$$

So, $2^{\frac{3}{2} k} \max _{i}\left|c_{k, i}\left(f^{\circ}\right)\right| \rightarrow 0$ and therefore $f^{\circ} \equiv$ const.

For any dyadic square $\Delta_{k, i} \times \Delta_{k, j}$ we can analogously consider the "vertical average" function of $f$ on this square and show that it is constant.

If there are $x_{1}, x_{2}, y_{0} \in[0,1], x_{1} \neq x_{2}$, such that $f\left(x_{1}, y_{0}\right) \neq f\left(x_{2}, y_{0}\right)$, then

$$
\int_{\Delta_{k, j}} f\left(x_{1}, y\right) d y \neq \int_{\Delta_{k, j}} f\left(x_{2}, y\right) d y
$$

for some small interval $\Delta_{k, j} \ni y_{0}$. But the function $v(x)=\int_{\Delta_{k, j}} f(x, y) d y$ is continuous, and we have proven that it is constant on each $\Delta_{k, i}$. Hence, it is constant on $[0,1]$, but this contradicts (1). 
So, $c^{(1)}$ - and $c^{(2)}$-coefficients of continuous functions cannot decay very fast in nontrivial sutiations. What can one say about $c^{(3)}$-coefficients? The main result of this paper is a rather surprising example of a function with identically zero $c^{(3)}$-coefficients.

Let us formulate our theorem. $\operatorname{By} \operatorname{Lip}(\alpha)$ we denote the space of functions $f:[0,1]^{2} \rightarrow \mathbb{R}$ such that the inequality

$$
\left|f(x, y)-f\left(x^{\prime}, y^{\prime}\right)\right| \leq C\left(\left|x-x^{\prime}\right|+\left|y-y^{\prime}\right|\right)^{\alpha},(x, y),\left(x^{\prime}, y^{\prime}\right) \in[0,1]^{2}
$$

holds for some $C=C(f)$.

Theorem 1. There exists a continuous function $f^{*}:[0,1]^{2} \rightarrow \mathbb{R}$ which is not represented as $f^{*}(x, y)=g(x)+h(y)$, such that $c_{k, i, j}^{(3)}\left(f^{*}\right)=0$ for all $k, i, j$. Moreover, $f^{*}$ lies in $\operatorname{Lip}(0.64)$.

We do not know the maximum possible smoothness of such functions. In particular, it would be interesting to construct a Lip(1) example of $f^{*}$, or to prove that it does not exist. One can see that there are no "simple" examples:

- the $c^{(3)}$-coefficients of a $C^{2}$-smooth function $f(x, y) \neq g(x)+h(y)$ cannot decay as $o\left(8^{-k}\right)$ :

$$
\varlimsup_{k \rightarrow \infty} 8^{k} \max _{i, j}\left|c_{k, i, j}^{(3)}(f)\right|>0
$$

- the $c^{(3)}$-coefficients of a piecewise-linear ${ }^{1}$ function $f(x, y) \neq g(x)+$ $+h(y)$ cannot decay as $o\left(4^{-k}\right)$.

Let us prove this. If $f \in C^{2}[0,1]^{2}$, then $\frac{\partial^{2} f}{\partial x \partial y} \neq 0$ in some point (otherwise $f(x, y)$ is represented as $g(x)+h(y))$. So, $\left|\frac{\partial^{2} f}{\partial x \partial y}\right| \geq c_{0}>0$ in some neighbourhood of that point. Using that $c_{k, i, j}^{(3)}(f)=2^{-3 k-4} \cdot \frac{\partial^{2} f}{\partial x \partial y}(\xi)$ for some $\xi \in \Delta_{k, i} \times \Delta_{k, j}$, we obtain (2).

Consider a piecewise-linear function: the square $[0,1]^{2}$ is triangulated, $f$ is linear on each triangle. One can show that there exists two triangles $A B C$ and $A B D$, having a common side $A B$, which is not vertical nor

${ }^{1}$ We say that a function $f:[0,1]^{2} \rightarrow \mathbb{R}$ is piecewise linear, if $f$ is continuous on $[0,1]^{2}$ and for some triangulation of the square, $f$ is linear on each triangle. 
horizontal, such that the linear functions corresponding to $A B C$ and $A B D$ differ. We can subtract a linear function from $f$ (this does not change $c^{(3)}$ ) and make it equal to zero on the triangle $A B C$. Moreover, we normalize $f$ to ensure that $(\partial f / \partial x)^{2}+(\partial f / \partial y)^{2} \equiv 1$ on $A B D$. For any sufficiently large $k$ there exists a dyadic square $\Delta_{k, i} \times \Delta_{k, j}$, which intersects $A B$ and does not intersect any triangles other that $A B C, A B D$. One can prove that $c_{k, i, j}^{(3)}(f) \neq 0$ in this "standard" situation 2 It follows (using the compactness argument and scaling) that $\max _{i, j}\left|c_{k, i, j}^{(3)}(f)\right| \geq R \cdot 4^{-k}$ for large $k$, where the constant $R$ depends only on the slope of the segment $A B$.

The next two sections are devoted to the proof of our theorem.

\section{Construction of the function $f^{*}$.}

Proof of Theorem 1. We consider linear functions

$$
L(x, y)=A x+B y+C \quad \text { such that } \iint_{[0,1]^{2}} L(x, y) d x d y=0 .
$$

It is clear that the function $L(x, y)$ from (3) with $L(0,0)=a, L(0,1)=b$, $L(1,1)=c, L(1,0)=d$ exists if and only if $a+c=b+d=0$. We denote such (unique) function as $L_{a, b}$; it is just $L_{a, b}(x, y)=a-(a+b) x-(a-b) y$. These functions will be the "building blocks" of our construction. Note that always $c_{k, i, j}^{(3)}\left(L_{a, b}\right)=0$.

For $b \in \mathbb{R}, b \neq 1$, we consider the space $C_{b}$ of functions $f:[0,1]^{2} \rightarrow \mathbb{R}$ with the following properties:

(i) $f$ is continuous on $[0,1]^{2}$;

(ii) $f(0,0)=1, f(0,1)=b, f(1,1)=1, f(1,0)=b$;

(iii) $f$ is linear on the sides of $[0,1]^{2}$;

(iv) $\iint_{[0,1]^{2}} f(x, y) d x d y=0$.

Suppose that for some $t, \lambda_{i}, u_{i} \in \mathbb{R}(i=1,2,3,4)$ the following equalities hold:

$$
\left(1, \frac{b+1}{2}, t, \frac{b+1}{2}\right)=\lambda_{1}(1, b, 1, b)+\lambda_{2}(b, 1, b, 1)+\left(u_{1}, u_{2},-u_{1},-u_{2}\right),
$$

${ }^{2}$ It is sufficient to prove that $c_{0,1,1}(f) \neq 0$ for $f(x, y)=\max (0, a x+b y+c)$ if line $a x+b y+c=0$ intersects the interior of $[0,1]^{2}$. This can be done by considering different cases of position of line $a x+b y+c=0$. 


$$
\left(\frac{b+1}{2}, b, \frac{b+1}{2}, t\right)=\lambda_{3}(1, b, 1, b)+\lambda_{4}(b, 1, b, 1)+\left(u_{3}, u_{4},-u_{3},-u_{4}\right) .
$$

Then we can define the operator $T: C_{b} \rightarrow C_{b}$ by the following rules:

$$
\begin{gathered}
T f\left(\frac{x}{2}, \frac{y}{2}\right)=T f\left(1-\frac{x}{2}, 1-\frac{y}{2}\right)=\lambda_{1} f(x, y)+\lambda_{2} f(1-x, y)+L_{u_{1}, u_{2}}(x, y), \\
T f\left(\frac{x}{2}, \frac{1+y}{2}\right)=T f\left(1-\frac{x}{2}, \frac{1-y}{2}\right)= \\
=\lambda_{3} f(x, y)+\lambda_{4} f(1-x, y)+L_{u_{3}, u_{4}}(x, y) .
\end{gathered}
$$

Let us show that $T f$ indeed lies in $C_{b}$. First, note that properties (ii) and (iii) of $C_{b}$ imply that $f(0,1 / 2)=f(1 / 2,1)=f(1,1 / 2)=f(1 / 2,0)=$ $=(b+1) / 2$ and the same should hold for $T f$. Consider the definition of $T f$ on the square $[0,1 / 2]^{2}$. The equality (4) implies that

$$
(T f(0,0), T f(0,1 / 2), T f(1 / 2,1 / 2), T f(1 / 2,0))=\left(1, \frac{b+1}{2}, t, \frac{b+1}{2}\right) \text {. }
$$

In particular, $t=T f(1 / 2,1 / 2)$. Definition for other quater-squares is similar. Note that the values of $T f$ on common vertices of that squares agree. Now we check properties (i)-(iv) of $T f$ : (ii) follows from $(6)$ and analogous equalities; properites (iii) and (iv) follow from the corresponding properties of $f$ and $L_{a, b}$. Finally, $T f$ is continuous because values of $T f$ on different quarter-squares are continuously "glued" by linearity of $T f$ on the sides of all that squares.

Soon we will show that if

$$
\varkappa:=\max \left(\left|\lambda_{1}\right|+\left|\lambda_{2}\right|,\left|\lambda_{3}\right|+\left|\lambda_{4}\right|\right)<1,
$$

then $T$ is a contraction (say, in the uniform metric). We claim that one can take $f^{*}$ to be the fixed point of $T$. Indeed, $f^{*}$ is not represented as $g(x)+h(y)$ because $f(0,0)+f(1,1) \neq f(0,1)+f(1,0)$ (recall that $b \neq 1)$. Next, $f^{*}$ is continuous by construction. Finally, we check that $c_{k, i, j}^{(3)}\left(f^{*}\right)=0$ using induction on $k$. If $k=0$, it follows from the fact that the average of $f^{*}$ on $[0,1]^{2}$ is zero and hence it is zero on all quartersquares. Suppose that $c_{k-1, i, j}^{(3)}\left(f^{*}\right)=0$ for all $i, j$. Note that $c_{k, i, j}^{(3)}\left(L_{a, b}\right)=$ $=0$. So, if $1 \leq i, j<2^{k-1}$, we have

$$
c_{k, i, j}^{(3)}\left(f^{*}\right)=c_{k, i, j}^{(3)}\left(\lambda_{1} f^{*}(2 x, 2 y)+\lambda_{2} f^{*}(1-2 x, 2 y)+L_{u_{1}, u_{2}}(2 x, 2 y)\right)=
$$




$$
=\frac{1}{2}\left(\lambda_{1} c_{k-1, i, j}^{(3)}\left(f^{*}\right)+\lambda_{2} c_{k-1,2^{k-1}-i+1, j}^{(3)}\left(f^{*}\right)\right)=0 .
$$

Other cases are analogous.

Here is a proof-of-concept simple example of configuration for $T$ with $\varkappa=3 / 4: b=0, t=-\frac{1}{2},\left(\lambda_{i}\right)=\left(\frac{1}{4}, \frac{1}{2}, \frac{1}{2},-\frac{1}{4}\right),\left(u_{i}\right)=\left(\frac{3}{4}, 0,0, \frac{1}{4}\right)$.

3. Contractivity of $T$. Recall that we want to prove more than just the continuity of $f^{*}$, but also some smoothness. Therefore we will work in the space $C_{b} \cap \operatorname{Lip}(\alpha)$ and find a fixed point $f^{*}$ of $T$ there. We need a convenient metric to prove contractivity of $T$. Denote by $S_{k, i, j}$ the square $\Delta_{k, i} \times \Delta_{k, j}$, and, as usual, $\omega(f, S)=\sup |f(A)-f(B)|$. It is $A, B \in S$

rather clear that a continuous functions belong to $\operatorname{Lip}(\alpha)$ if and only if $\max _{1 \leq i, j \leq 2^{k}} \omega\left(f, S_{k, i, j}\right)=O\left(2^{-k \alpha}\right)$. So, we take

$$
\rho(f, g)=\sup _{k \geq 0} 2^{k \alpha} \max _{1 \leq i, j \leq 2^{k}} \omega\left(f-g, S_{k, i, j}\right) .
$$

It is indeed a complete metric in $C_{b} \cap \operatorname{Lip}(\alpha)$. (Note that if $f-g \equiv$ const, $f, g \in C_{b}$, then $f \equiv g$.) Also, $T$ preserves space $C_{b} \cap \operatorname{Lip}(\alpha)$, as one can see, e.g., from (8).

Let us prove that

$$
\rho(T f, T g) \leq \varkappa 2^{\alpha} \rho(f, g),
$$

hence $T$ is a contraction provided $\varkappa<2^{-\alpha}$. We should estimate

$$
\omega\left(T f-T g, S_{k, i, j}\right) .
$$

Suppose that $S_{k, i, j} \subset[0,1 / 2]^{2}$. Then, by definition of $T$,

$$
\begin{aligned}
& \omega\left(T f-T g, S_{k, i, j}\right)= \\
& =\omega\left(\lambda_{1} f(2 x, 2 y)+\lambda_{2} f(1-2 x, 2 y)-\lambda_{1} g(2 x, 2 y)-\lambda_{2} g(1-2 x, 2 y), S_{k, i, j}\right) \leq \\
& \quad \leq\left|\lambda_{1}\right| \omega\left(f-g, S_{k-1, i, j}\right)+\left|\lambda_{2}\right| \omega\left(f-g, S_{k-1,2^{k-1}-i+1, j}\right) \leq \\
& \leq \varkappa 2^{-(k-1) \alpha} \rho(f, g) .
\end{aligned}
$$

Other cases are completely analogous. The claim (7) follows.

The last thing to do is to minimize $\varkappa$. One should use the following configuration ( $t$ and $u_{i}$ may be recovered using (4, 5)):

$b=\frac{-5+\sqrt{17}}{2}, \quad \lambda_{1}=\frac{1}{4}, \quad \lambda_{2}=\frac{-1+\sqrt{17}}{8}, \quad \lambda_{3}=0, \quad \lambda_{4}=\frac{-1-\sqrt{17}}{8}$. 
This gives $\varkappa=(1+\sqrt{17}) / 8=0.6403 \ldots$ and $\alpha<-\log _{2} \varkappa=0.6429 \ldots$ The theorem is proven.

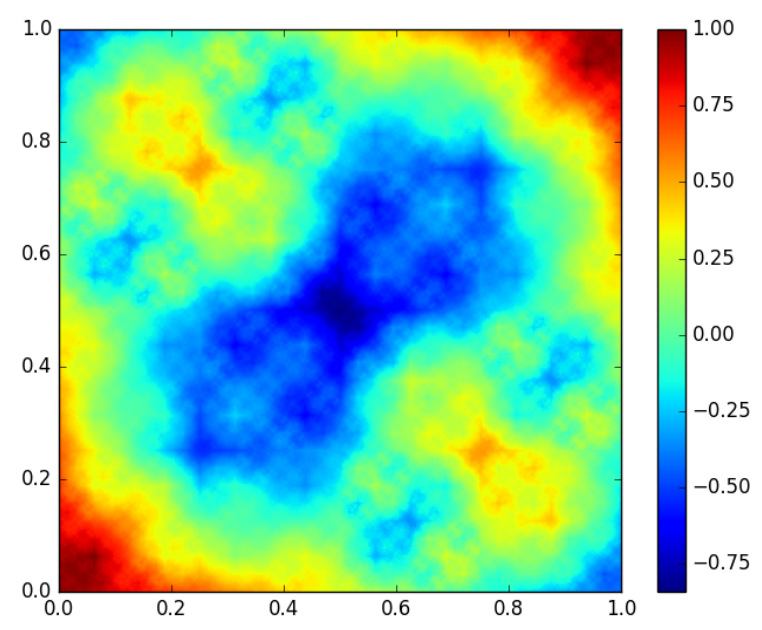

Fig. 2. Function $f^{*}$

\section{References}

[1] Kashin B. S., Sahakyan A. A. Orthogonal series. American Mathematical Soc., 2005, 451 p.

[2] Novikov I. Ya., Protasov V. Yu., Skopina M. A. Wavelet theory. American Mathematical Soc., 2011, 506 p.

Received April 15, 2017.

In revised form, June 15, $201 \%$.

Accepted June 19, 2017.

Published online June 21, $201 \%$.

Petrozavodsk State University

33, Lenina pr., Petrozavodsk 185910, Russia

E-mail: elena.belkina@gmail.com

Steklov Mathematical Institute

8, Gubkina st., Moscow 119991, Russia

E-mail:malykhin@mi.ras.ru 